\title{
SPSS macros to compare any two fitted values from a regression model
}

\author{
Bruce Weaver • Sacha Dubois
}

Published online: 19 May 2012

(C) Psychonomic Society, Inc. 2012

\begin{abstract}
In regression models with first-order terms only, the coefficient for a given variable is typically interpreted as the change in the fitted value of $Y$ for a one-unit increase in that variable, with all other variables held constant. Therefore, each regression coefficient represents the difference between two fitted values of $Y$. But the coefficients represent only a fraction of the possible fitted value comparisons that might be of interest to researchers. For many fitted value comparisons that are not captured by any of the regression coefficients, common statistical software packages do not provide the standard errors needed to compute confidence intervals or carry out statistical tests-particularly in more complex models that include interactions, polynomial terms, or regression splines. We describe two SPSS macros that implement a matrix algebra method for comparing any two fitted values from a regression model. The !OLScomp and !MLEcomp macros are for use with models fitted via ordinary least squares and maximum likelihood estimation, respectively. The output from the macros includes the standard error of the difference between the two fitted
\end{abstract}

Electronic supplementary material The online version of this article (doi:10.3758/s13428-012-0204-2) contains supplementary material, which is available to authorized users.

B. Weaver $(\square)$

Human Sciences Division, Northern Ontario School of Medicine,

Thunder Bay, ON, Canada P7B 5E1

e-mail: bweaver@lakeheadu.ca

B. Weaver $\cdot$ S. Dubois

Centre for Research on Safe Driving, Lakehead University,

Thunder Bay, ON, Canada P7B 5E1

S. Dubois

St. Joseph's Care Group,

580 N. Algoma St.,

Thunder Bay, ON, Canada P7B 5G4 values, a 95\% confidence interval for the difference, and a corresponding statistical test with its $p$-value.

Keywords Regression · Ordinary least squares · Maximum likelihood estimation $\cdot$ SPSS

\section{SPSS macros to compare any two fitted values from a regression model}

Students are often introduced to regression models via ordinary least squares (OLS) linear regression, starting with the simple linear regression model. That model can be written as

$Y=a+b X+e$

where $Y$ is a continuous outcome (or dependent) variable, $X$ is a continuous explanatory (or predictor) variable, ${ }^{1} a$ is the constant (or intercept), $b$ is the slope of the linear relationship between $X$ and $Y$, and $e$ is the fitting error. It can also be written as

$Y^{\prime}=a+b X$

where $Y^{\prime}=Y-e=$ the fitted (or predicted) value of $Y$.

Students may then be introduced to the multiple linear regression model (with first-order terms only), ${ }^{2}$ which can be written as

$Y=a+b_{1} X_{1}+b_{2} X_{2}+\ldots+b_{p} X_{p}+e$

\footnotetext{
${ }^{1}$ As noted by one of the reviewers, $X$ can also be dichotomous. When $X$ is dichotomous, the simple linear regression model is equivalent to an independent groups $t$-test (equal variances version).

${ }^{2}$ Such models are sometimes described as main effects only models, but that terminology is technically incorrect because partial effects in a regression model are not the same thing as main effects in a factorial ANOVA model.
} 
with $p$ representing the number of predictor variables in the model. The multiple regression model can also be written as

$Y^{\prime}=a+b_{1} X_{1}+b_{2} X_{2}+\ldots+b_{p} X_{p}$

with $Y^{\prime}$ once again representing the fitted value of $Y$.

In any regression model, the constant $a$ gives the fitted value of $Y$ when all explanatory variables are equal to 0 . For the simple linear regression model (Eqs. 1 and 2), $b$ gives the change in the fitted value of $Y$ for a one-unit increase in $X$. For the multiple linear regression model with first-order terms only (Eqs. 3 and 4), letting $j$ range from 1 to $p, b_{j}$ gives the change in the fitted value of $Y$ for a one-unit increase in $X_{j}$ while controlling for all of the other explanatory variables in the model (i.e., while holding them all constant).

As the phrase "change in the fitted value of $Y$ " suggests, the $b$-coefficients from the single predictor model and the multiple regression model with no higher order terms represent the difference between two fitted values of $Y$. Starting with the single predictor model, let $Y_{X}^{\prime}$ be the fitted value of $Y$ when some value of $X$ is plugged into Eq. 2, and $Y_{X+1}^{\prime}$ the fitted value of $Y$ when $X+1$ is plugged into Eq. 2:

$Y_{X}^{\prime}=a+b X$

$Y_{(X+1)}^{\prime}=a+b(X+1)=a+b X+b$

Subtracting Eq. 5 from Eq. 6 results in $b$, representing the change in the fitted value of $Y$ for a one-unit increase in $X$ (see Eq. 7):

$$
\begin{aligned}
& Y_{X+1}^{\prime}-Y_{X}^{\prime}=(a+b X+b)-(a+b X) \\
& \quad=a+b X+b-a-b X=b
\end{aligned}
$$

The coefficients for a multiple regression model (with first-order terms only) can be obtained in the same manner. For example, in a model with two explanatory variables, three different fitted values of $Y$ can be obtained as follows:

$Y_{X_{1}, X_{2}}^{\prime}=a+b_{1} X_{1}+b_{2} X_{2}$

$Y_{X_{1}+1, X_{2}}^{\prime}=a+b_{1}\left(X_{1}+1\right)+b_{2} X_{2}$

$$
=a+b_{1} X_{1}+b_{2} X_{2}+b_{1}
$$

$$
\begin{aligned}
Y_{X_{1}, X_{2}+1}^{\prime} & =a+b_{1} X_{1}+b_{2}\left(X_{2}+1\right) \\
& =a+b_{1} X_{1}+b_{2} X_{2}+b_{2}
\end{aligned}
$$

Subtracting Eq. 8 from 9 leaves $b_{1}$, which represents the change in the fitted value of $Y$ for a one-unit increase in $X_{1}$ with $X_{2}$ held constant. Similarly, subtracting Eq. 8 from 10 leaves $b_{2}$, which represents the change in the fitted value of $Y$ for a one-unit increase in $X_{2}$ with $X_{1}$ held constant.

Some users of regression models may think that the only fitted value comparisons available to them are the ones that correspond to regression coefficients. But in reality, a host of other fitted value comparisons are possible, and from a research or hypothesis-testing point of view, many of them might be more meaningful than the small subset of comparisons provided by the coefficients. For example, if $X_{1}$ and $X_{2}$ are positively correlated, it may not be very realistic to hold one of them constant while increasing the other by one unit. Rather, it might be more informative to determine the change in the fitted value of $Y$ when $X_{1}$ increases by 5 and $X_{2}$ increases by 10 . Eq. 11 shows the fitted value of $Y$ for $X_{1}+5$ and $X_{2}+10$ :

$$
\begin{aligned}
& Y_{X_{1}+5, X_{2}+10}^{\prime}=a+b_{1}\left(X_{1}+5\right)+b_{2}\left(X_{2}+10\right) \\
& \quad=a+b_{1} X_{1}+5 b_{1}+b_{2} X_{2}+10 b_{2}
\end{aligned}
$$

Subtracting Eq. 8 from Eq. 11 results in the weighted combination of two coefficients, $5 b_{1}+10 b_{2}$ (see Eq. 12):

$$
\begin{aligned}
& Y_{X_{1}+5, X_{2}+10}^{\prime}-Y_{X_{1}, X_{2}}^{\prime}=\left(a+b_{1} X_{1}+5 b_{1}+b_{2} X_{2}+10 b_{2}\right) \\
& -\left(a+b_{1} X_{1}+b_{2} X_{2}\right) \\
& =a+b_{1} X_{1}+5 b_{1}+b_{2} X_{2}+10 b_{2} \\
& -a-b_{1} X_{1}-b_{2} X_{2} \\
& =a-a+b_{1} X_{1}-b_{1} X_{1}+b_{2} X_{2} \\
& -b_{2} X_{2}+5 b_{1}+10 b_{2} \\
& =5 b_{1}+10 b_{2}
\end{aligned}
$$

Fitted value comparisons of interest may also entail weighted combinations of two or more coefficients in models that contain interactions, polynomial terms, or regression splines. For example, a few years ago, we fitted the logistic regression model shown in Eq. $13^{3}$ :

$Y^{\prime}=\ln \left(\frac{p}{1-p}\right)=a+b_{1} B A C+b_{2} B A C^{2}+$ other variables

In this example, the outcome variable was the presence of an unsafe driving action, and the main explanatory variable was blood alcohol concentration (BAC). But the inclusion of BAC-squared in the model made the relationship between $\mathrm{BAC}$ and the log-odds of the outcome curvilinear rather than linear. Because one of our colleagues wished to report odds ratios for BAC values of .05 and .08 , both relative to

\footnotetext{
${ }^{3}$ In Eq. 13, $p$ does not represent the number of outcome variables. Rather, it represents the probability that $Y$, the dichotomous outcome variable, is equal to 1 rather than 0 .
} 
$\mathrm{BAC}=0$, we made two fitted value comparisons involving Eqs. 14,15 , and 16 :

$Y_{B A C=0}^{\prime}=a+b_{1} 0+b_{2} 0^{2}+$ other variables

$Y_{B A C=0.05}^{\prime}=a+b_{1} 0.05+b_{2} 0.05^{2}+$ other variables

$Y_{B A C=0.08}^{\prime}=a+b_{1} 0.08+b_{2} 0.08^{2}+$ other variables

Subtracting Eq. 14 from 15 leaves $b_{1} \times 0.05+b_{2} \times 0.05^{2}$, and subtracting Eq. 14 from 16 leaves $b_{1} \times 0.08+b_{2} \times$ $0.08^{2}$. These two weighted combinations of $b_{1}$ and $b_{2}$ give the logarithms of the desired odds ratios: The odds ratio for $\mathrm{BAC}=0.05, \mathrm{OR}_{0.05}$, is equal to $\operatorname{Exp}\left(b_{1} \times 0.05+b_{2} \times 0.05^{2}\right)$, and $\mathrm{OR}_{0.08}=\operatorname{Exp}\left(b_{1} \times 0.08+b_{2} \times 0.08^{2}\right)$.

In order to compute a $95 \%$ confidence interval for one of these fitted value comparisons, or to test whether the difference between the two fitted values is statistically significant, we need the standard error of the difference between the two fitted values. When a fitted value comparison of interest is captured by one of the regression coefficients, the needed standard error is reported in the regression output, as are the 95\% confidence interval and the corresponding statistical test. But in situations like the two described above, the desired fitted value comparison is not captured by any of the regression coefficients, and therefore, the needed standard error is not reported in the regression output. That standard error must be computed using a rather complicated formula involving variances and covariances. The two SPSS macros described in this article implement that formula.

\section{The !OLScomp and !MLEcomp macros}

In the remainder of this article, we describe two SPSS macros for comparing any two fitted values from a regression model and demonstrate their use. ${ }^{4}$ The !OLScomp and !MLEcomp macros are for use with regression models fitted by ordinary least squares (OLS) and maximum likelihood estimation (MLE), respectively. ${ }^{5}$ Both macros implement the matrix algebra method described by Johnson and

\footnotetext{
${ }^{4}$ The use of SPSS macros requires some knowledge of SPSS syntax. Readers who are not familiar with SPSS syntax may find the following tutorials helpful: http://www.spsstools.net/LearningSyntax.htm; http:// www.lrz.de/ wlm/ein spss.htm. A good introduction to SPSS macros can be found here: http://www.spsstools.net/MacroTutorial.htm.

${ }^{5}$ Linear regression and related models (e.g., ANOVA, ANCOVA) are typically fitted using OLS. Logistic regression and Poisson regression are typical examples of models fitted via MLE. The macro definitions for !OLScomp and !MLEcomp can be seen in SPSS syntax file OLScomp_and_MLEcomp_macros.SPS, which is available as part of the online Supplementary Material. Other syntax files available there contain demonstrations of how to use the macros.
}

Wichern (2002, p. 77, Equation 2-43). ${ }^{6}$ However, they differ somewhat in terms of macro arguments and the type of data file that is required as input.

\section{The !OLScomp macro}

The !OLScomp macro works on the same raw data file used to run the regression model of interest. It requires the following input in the form of macro arguments:

- $Y=$ the outcome variable for the regression model.

- $\mathrm{XList}=\mathrm{a}$ list of explanatory variables for the regression model.

- $\operatorname{Set}_{1}=$ a column vector of values plugged into the regression equation to compute the first fitted value of $Y$.

- $\mathrm{Set}_{2}=$ a column vector of values plugged into the regression equation to compute the second fitted value of $Y$.

- Title $=$ a title to be displayed on the output from the macro.

The outcome variable handed to the macro as the $\mathrm{Y}$ argument is read into a column vector called $Y$. The XList variables are included in a design matrix called $X$, the first column of which is a vector of $1 \mathrm{~s}$. This means that the constant is included when !OLScomp runs the regression model. With the $Y$ and $X$ matrices in hand, the macro then uses the usual matrix equation to compute $B$, a column vector of regression coefficients (see Eq. 17). ${ }^{7}$ After some intermediate computations to work out the $M S_{\text {error }}$ for the regression model, the macro computes $\operatorname{Cov} B$, the covariance matrix for the coefficients (Eq. 18). ${ }^{8}$

$B=\left(X^{T} X\right)^{-1} \mathrm{X}^{\mathrm{T}} \mathrm{Y}$

$\operatorname{Cov} B=\left(X^{T} X\right)^{-1} M S_{\text {error }}$

\footnotetext{
${ }^{6}$ We thank Ray Koopman (personal communication) for alerting us to this method.

${ }^{7}$ In Eq. 17 and subsequent matrix equations, the superscript " $T$ " means "transpose." For example, $X^{\mathrm{T}}$ is the transpose of matrix $X$. Some algebra textbooks use a prime rather than a superscript $\mathrm{T}$ - for example, $X^{\prime}$. But since we have already used $Y^{\prime}$ to represent the fitted value of $Y$ in a regression model, we think it will be less confusing for readers to use a superscript $\mathrm{T}$ to indicate transposition of a matrix. The superscript " -1 " indicates matrix inversion. We use the SPSS matrix language function $\operatorname{GINV}()$, rather than $\operatorname{INV}()$, to compute the inverse of a matrix. GINV() gives the Moore-Penrose generalized inverse. By chance, we discovered that our Example 1 model would not run using INV() unless we rescaled the explanatory variable, but it does run using GINV(). Readers who have SPSS can find more information in the Command Syntax Reference Manual by looking under MATRIXEND MATRIX $\rightarrow$ COMPUTE Statement $\rightarrow$ Matrix Functions. Readers who do not have SPSS can go to http://publib.boulder.ibm.com/ infocenter/spssstat/v20r0m0/index.jsp and search on INV and GINV.

${ }^{8}$ Equations 17 and 18 can be found in multiple references-for example, Fox (2008, p. 196).
} 
For the Set ${ }_{1}$ and Set ${ }_{2}$ arguments handed to the macro, the first value is an indicator for inclusion of the constant in the regression equation $(1=$ include the constant, $0=$ exclude the constant) and should typically be set equal to $1 .{ }^{9}$ The other values in $\mathrm{Set}_{1}$ and $\mathrm{Set}_{2}$ are either indicators for categorical variables or actual values of quantitative explanatory variables. As will be seen in the examples to follow, the Set $_{1}$ and $\mathrm{Set}_{2}$ vectors must be enclosed in braces (i.e., \{\} ) with the elements separated by semicolons. (In the SPSS matrix language, matrices are enclosed in braces, and semicolons indicate the end of a row.)

The following matrix algebra computations are then carried out:

$\operatorname{Diff}_{1}=$ Set $_{1}-$ Set $_{2}$

$\operatorname{Diff}_{2}=$ Set $_{2}-$ Set $_{1}$

$\mathrm{FV}_{1}=$ the first fitted value of $Y$ using Set $_{1}$ values $=B^{T}$ Set $_{1}$

$\mathrm{FV}_{2}=$ the second fitted value of $Y$ using Set $_{2}$ values $=B^{T}$ Set $_{2}$

$\mathrm{FV}_{1}-\mathrm{FV}_{2}=$ first fitted value minus second fitted value $=B^{T}$ Diff $_{1}$

$\mathrm{FV}_{2}-\mathrm{FV}_{1}=$ second fitted value minus first fitted value $=B^{T} \operatorname{Diff}_{2}$

$\operatorname{SE}\left(\mathrm{FV}_{1}\right)=$ standard error of $\mathrm{FV}_{1}=\sqrt{\operatorname{Set}_{1}^{\mathrm{T}} \times \operatorname{Cov} B \times \operatorname{Set}_{1}}$

$S E\left(\mathrm{FV}_{2}\right)=$ standard error of $\mathrm{FV}_{2}=\sqrt{\operatorname{Set}_{2}^{\mathrm{T}} \times \operatorname{Cov} B \times \mathrm{Set}_{2}}$

$S E\left(\mathrm{FV}_{1}-\mathrm{FV}_{2}\right)=\sqrt{\operatorname{Diff}_{1}^{\mathrm{T}} \times \operatorname{Cov} B \times \operatorname{Diff}_{1}}$

$S E\left(\mathrm{FV}_{2}-\mathrm{FV}_{1}\right)=\sqrt{\operatorname{Diff}_{2}^{\mathrm{T}} \times \operatorname{Cov} B \times \operatorname{Diff}_{2}}$

\footnotetext{
${ }^{9}$ When the macros are used to compare two fitted values of $Y$, the first variable in Set $_{1}$ and Set $_{2}$ must be set to 1 . But when the macros are used to examine a difference of differences, as in Example 3, the first variable may have to be set to 0 instead.
}

Finally, 95\% confidence intervals and $t$-tests are computed using the usual methods (see Appendix 1 for details).

\section{The !MLEcomp macro}

As was noted earlier, the !OLScomp macro computes the OLS regression model via the usual matrix algebra equations. Therefore, it is able to internally generate the $B$ and $\operatorname{Cov} B$ matrices that are needed to compute the desired standard errors, confidence intervals, and statistical tests. But that approach will not work for !MLEcomp, because maximum likelihood estimation is an iterative process, and the regression equation cannot be obtained by solving some matrix equations. Therefore, unlike !OLScomp, !MLEcomp does not use a raw data file as input. Rather, it requires an input file that contains both $B$, the vector of regression coefficients, and $\operatorname{Cov} B$, the covariance matrix for $B$. This means that users of !MLEcomp must first run their model using a standard SPSS procedure (e.g., GENLIN) and have it save $B$ and $\operatorname{Cov} B$. Some data management is then required, but it is not too onerous. An example is provided in the online Supplementary Material (see syntax file Example_2.sps). ${ }^{10}$ Note that the variable containing the regression coefficients must be named $\mathrm{B}$ and the variables making up $\operatorname{Cov} B$ must be contiguous and in the same order as the coefficients.

The macro arguments for !MLEcomp are as follows:

- FirstCovB $=$ the variable name for the first column of CovB

- $\quad$ LastCovB $=$ the variable name for the last column of CovB

- Set $_{1}$ and $\mathrm{Set}_{2}$, the values that must be plugged into the regression equation to compute the first and second fitted values

- $\operatorname{ExpB}=$ an indicator $(1=$ Yes, $0=$ No $)$ for exponentiation of the fitted value and its confidence limits (used for !MLEcomp only) $)^{11}$

- Title $=$ a title to appear on output from !MLEcomp.

\footnotetext{
${ }^{10}$ Please note that some of the data management steps contained in this syntax file operate on data sets generated via the OMS command. Variable names created by OMS depend on the output language that is in effect. For example, with English as the output language, our OMS command generates a variable called "Intercept"; but with Spanish as the output language, the same variable is named "Intersección." In order to avoid this difference, which would cause an error, we issue a command near the top of the syntax file to set the output language to English. Users of non-English versions will have to reset to their preferred output language after running our syntax file. In order to assist users who are not comfortable using syntax for data management, we also include a list of steps that can be carried out manually to create the needed BcovB data set.

${ }^{11}$ For models that use log or logit transformations, it is customary to exponentiate the regression coefficient. In logistic regression, for example, $\operatorname{Exp}(\mathrm{B})=e^{B}=$ an odds ratio.
} 
!MLEcomp then carries out the computations shown in Eqs. 19, 20, 21, 22, 23, 24, 25, 26, 27, and 28. Whereas !OLScomp computes $t$-tests, !MLEcomp computes Wald tests (see Appendix 1 for details). And finally, if the ExpB argument is set to 1, !MLEcomp also exponentiates $\mathrm{FV}_{1}$, $\mathrm{FV}_{2}, \mathrm{FV}_{1}-\mathrm{FV}_{2}, \mathrm{FV}_{2}-\mathrm{FV}_{1}$ and the limits of their respective confidence intervals.

\section{Examples}

We now turn to some examples that illustrate the use of both !OLScomp and !MLEcomp in situations where the fitted value comparisons of interest do not correspond to coefficients reported in the regression output.

Example 1: A linear regression model with a quadratic term

The first example uses a linear regression model with $Y=$ miles per gallon (MPG) and $X=$ vehicle weight in pounds. ${ }^{12}$ Because the relationship is curvilinear (see Fig. 1), weightsquared was also included in the model. The SPSS syntax shown in Syntax Box 1 was used to run the model via the REGRESSION procedure.

Syntax Box 1. SPSS syntax to run the linear regression model for Example 1

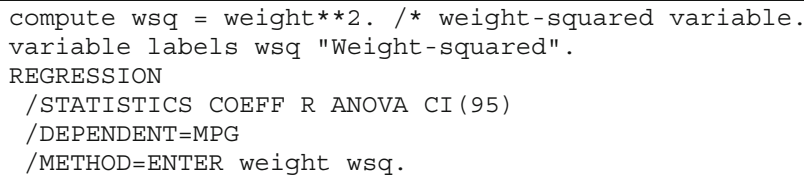

The model is statistically significant, $F(2,394)=$ 489.123, $p<.001$; and $R^{2}=.713$. As was expected, the regression coefficients for weight and weight-squared are both statistically significant (see Table 1). The significance of the weight-squared term indicates that the effect (on MPG) of increasing vehicle weight by a given amount depends on how heavy the vehicle is. This dependency can be illustrated by making the following comparisons: 3,000 lbs-2,000 lbs, 4,000-3,000, and 5,000-4,000.

Syntax Box 2 shows how to make the first of those three fitted value comparisons using !OLScomp. As was noted earlier, the first value in the Set $_{1}$ and Set $_{2}$ vectors is an indicator for inclusion of the constant (or intercept) in the

\footnotetext{
$\overline{12}$ All examples in this article use sample data files that come with SPSS. (For readers who may not have access to them, the data files are also available as part of the online Supplementary Material.) These examples are intended only to illustrate the use of the !OLScomp and !MLEcomp macros. Substantive conclusions about the subject matter of the examples are unwarranted and drawn at the reader's own risk. Example 1 uses data file Cars.sav file. Note that one odd case with vehicle weight less than $1,000 \mathrm{lbs}$ was excluded.
}

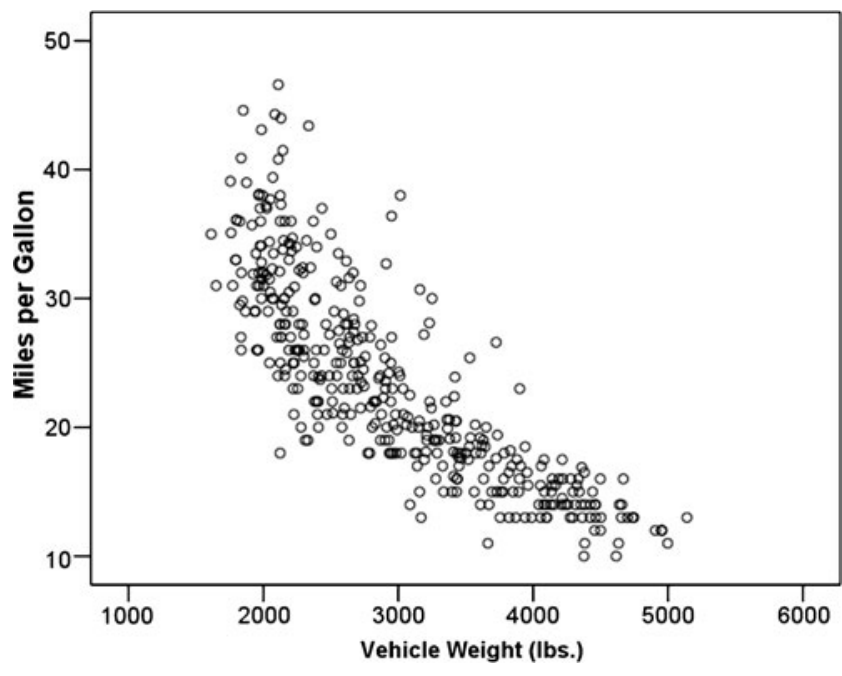

Fig. 1 Miles per gallon as a function of vehicle weight

model $(1=$ include, $0=$ exclude $)$. It must be set to 1 in this case, because the two fitted values being compared were derived from a model that includes the constant. For this example, the second value is vehicle weight, and the third value is weight-squared. Note that rather than entering the actual value of weight squared, one can use "**," which is the exponentiation operator in SPSS: "**2" means to the power of 2 (or squared), “**3" means to the power of 3 (cubed), and so on. In the example below, $3,000 * * 2=$ $3,000^{2}=9,000,000$; and $2,000 * * 2=2,000^{2}=4,000,000$.

Syntax Box 2. !OLScomp syntax to compare fitted values of MPG for vehicles weighing $3,000 \mathrm{lbs}$ versus $2,000 \mathrm{lbs}$

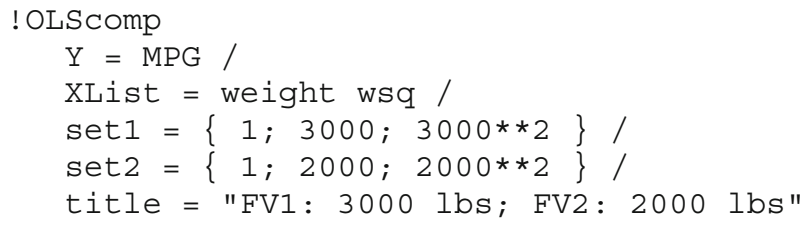

Table 1 Regression coefficients for Example 1

\begin{tabular}{|c|c|c|c|c|c|c|c|}
\hline \multicolumn{8}{|l|}{ Coefficients $^{\mathrm{a}}$} \\
\hline \multirow[t]{2}{*}{ Model } & \multicolumn{2}{|c|}{$\begin{array}{l}\text { Unstandardized } \\
\text { coefficients }\end{array}$} & \multirow{2}{*}{$\begin{array}{l}\text { Standardized } \\
\text { coefficients } \\
\text { Beta }\end{array}$} & \multirow[t]{2}{*}{$\mathrm{t}$} & \multirow[t]{2}{*}{ Sig. } & \multicolumn{2}{|c|}{$\begin{array}{l}95.0 \% \text { Confidence } \\
\text { interval for B }\end{array}$} \\
\hline & B & Std. error & & & & $\begin{array}{l}\text { Lower } \\
\text { bound }\end{array}$ & $\begin{array}{l}\text { Upper } \\
\text { bound }\end{array}$ \\
\hline 1 (Constant) & 62.762 & 2.984 & & 21.033 & .000 & 56.895 & 68.629 \\
\hline $\begin{array}{l}\text { Vehicle Weight } \\
\text { (lbs) }\end{array}$ & -.019 & .002 & -2.037 & -9.557 & .000 & -.023 & -.015 \\
\hline Weight-squared & $1.749 \mathrm{E}-6$ & .000 & 1.217 & 5.709 & .000 & .000 & .000 \\
\hline
\end{tabular}

a. Dependent Variable: Miles per Gallon 
Output Box 1. Output from the !OLScomp command shown in Syntax Box 2

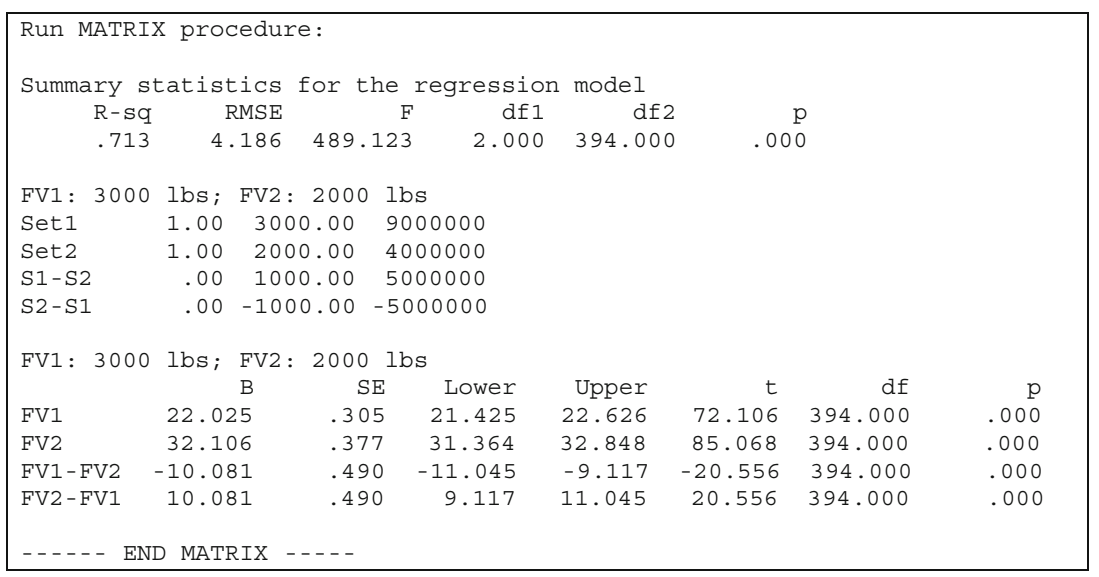

Output from the !OLScomp command in Syntax Box 2 is shown in Output Box 1. The output is organized into three sections. The first section shows the $R^{2}$ value for the model and the omnibus $F$-test. These values can be compared with the output generated via the REGRESSION procedure (or UNIANOVA, etc.) to verify that !OLScomp has run the same model.

The second part of the output shows the $\mathrm{Set}_{1}$ and $\mathrm{Set}_{2}$ values that were handed to !OLScomp along with the Set $_{1}-$ $\mathrm{Set}_{2}$ and $\mathrm{Set}_{2}-\mathrm{Set}_{1}$ differences. Note that even though the values were $3000 * * 2$ and $2000 * * 2$ when the macro was called, they appear as 9000000 and 4000000, respectively, in the output.

The final part of the output shows the two fitted values $\left(F_{1}\right.$ and $\left.F_{2}\right)$ along with the differences between them $\left(\mathrm{FV}_{1}-\mathrm{FV}_{2} \text { and } \mathrm{FV}_{2}-\mathrm{FV}_{1}\right)^{13}$

- $\mathrm{FV}_{1}=22.025=$ fitted MPG for a 3,000 lb vehicle

- $\mathrm{FV}_{2}=32.106=$ fitted MPG for a 2,000 $\mathrm{lb}$ vehicle

- $\mathrm{FV}_{1}-\mathrm{FV}_{2}=22.025-32.106=-10.081$

- $\mathrm{FV}_{2}-\mathrm{FV}_{1}=32.106-22.025=10.081$.

Importantly, the standard errors and 95\% confidence intervals are also reported for each of these estimates, as are $t$-tests and $p$-values. The null hypothesis for each of the $t$-tests states that the parameter being tested (i.e., the fitted

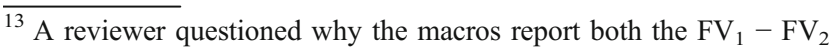
and $\mathrm{FV}_{2}-\mathrm{FV}_{1}$ differences, given that they always have the same absolute value and the same standard error. We report both so that if a user decides after the fact that $F_{2}-F_{1}$ is more easily interpreted than $\mathrm{FV}_{1}-\mathrm{FV}_{2}$, he or she is spared the trouble of having to rerun the macro with Set1 and Set2 swapped, or to compute the confidence interval by hand (which would needlessly introduce a possible source of error). This method of reporting is also consistent with how SPSS reports pairwise comparisons of means via EMMEANS with the COMPARE option.
}

value, or the difference between two fitted values) is equal to zero.

!OLScomp syntax to make the other two comparisons of interest $(4,000-3,000$ and 5,000-4,000) is shown in Syntax Box 3. The corresponding output is shown in Output Box 2.

Note that for each of the three analyses (Output Boxes 1 and 2), the $\mathrm{FV}_{1}-\mathrm{FV}_{2}$ difference is negative, which means that MPG is lower for the heavier vehicle in each case, as was expected. But note that even though the difference in vehicle weight is $1,000 \mathrm{lbs}$ in every case, the effect of a $1,000-\mathrm{lb}$ change is not constant. This is because of the curvilinear relationship between weight and MPG (see Fig. 1). One way to think of the weight-squared term in the model is that weight interacts with itself. In other words, the effect of increasing vehicle weight by 1,000 lbs depends on the value of weight. As the results from !OLScomp show, increasing weight from 2,000 to 3,000 leads to a drop of about $10 \mathrm{MPG}$, whereas increasing weight from 4,000 to 5,000 leads to a drop of only about 3 MPG.

Syntax Box 3. ! OLScomp syntax to compare fitted values of MPG for vehicles weighing 4,000 lbs versus $3,000 \mathrm{lbs}$ and 5,000 lbs versus $4,000 \mathrm{lbs}$

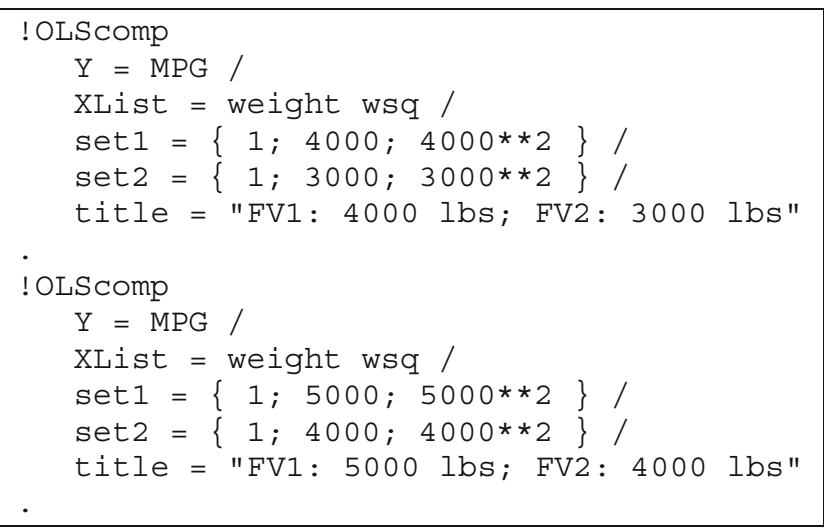


Output Box 2. Output from the !OLScomp commands shown in Syntax Box 3

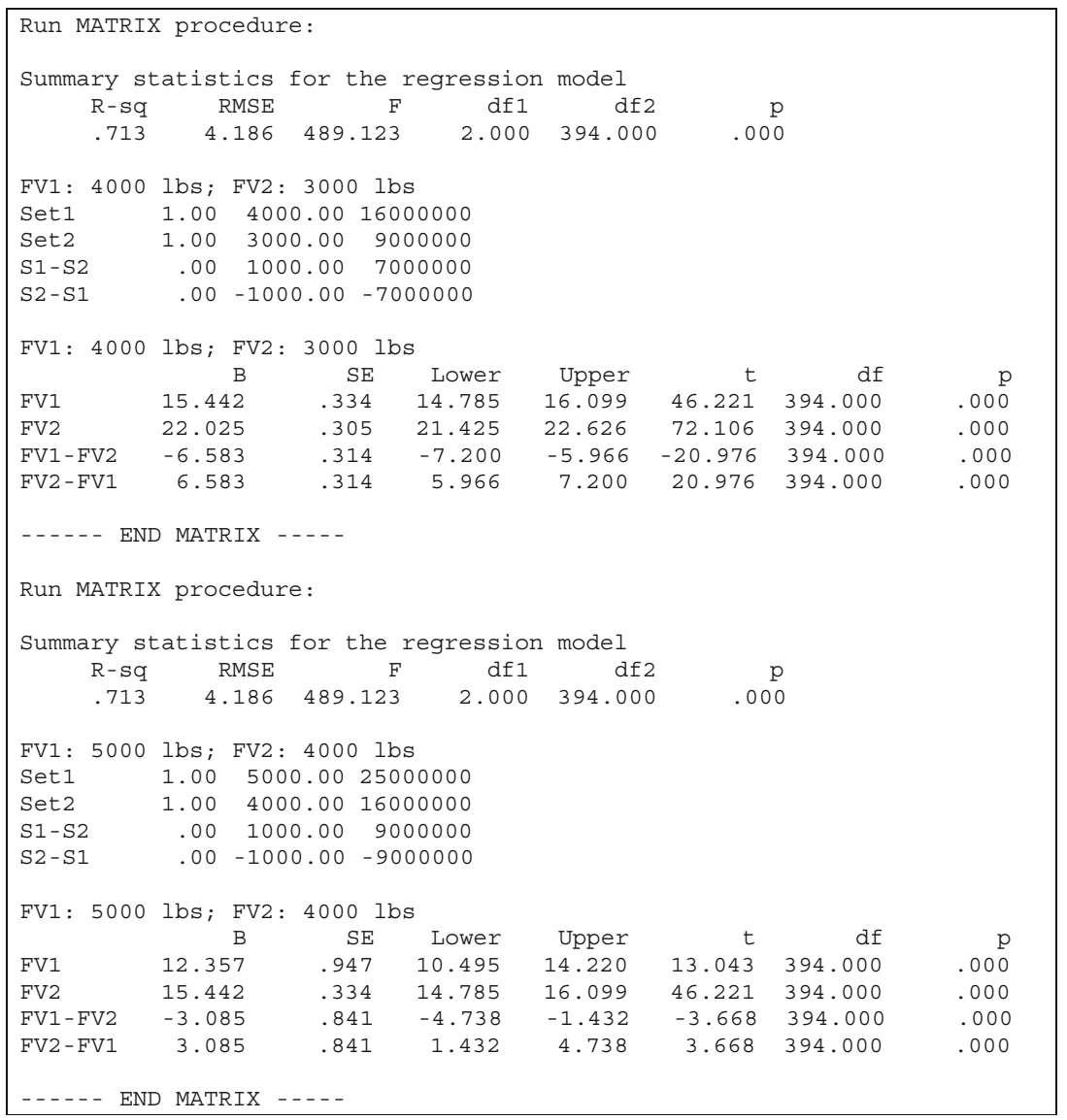

Example 2: Logistic regression with a categorical $\times$ continuous interaction

This second example demonstrates use of the !MLEcomp macro. We use a logistic regression model with education level, number of years with current employer, and the interaction between them as predictors of defaulting on a bank loan. The data are from the bankloan.sav sample data file that comes with SPSS. For education level, the ED variable in the data file was recoded into a new variable (EDUC) with the college grad and postgraduate degree categories combined. This was done because the number of cases in the postgraduate degree category was very low $(n=5$ out of 700 total cases). The model was run via the GENLIN command shown in Syntax Box 4. ${ }^{14}$ The OMS commands ${ }^{15}$

\footnotetext{
${ }^{14}$ We used GENLIN because it produces a covariance matrix for the coefficients that is easier to work with than the one provided by the LOGISTIC REGRESSION procedure. However, we also ran the model via LOGISTIC REGRESSION, because (unlike GENLIN) it provides multiple degree of freedom tests for categorical explanatory variables with two or more levels.

${ }^{15}$ OMS stands for output management system. It allows one to direct output that would ordinarily appear in the output viewer (e.g., tables of regression coefficients) to a data file for further processing.
}

were included to write the regression coefficients to a new data set called " $\mathrm{B}$ " and the covariance matrix for $\mathrm{B}$ to a new data set called "covB."

Syntax Box 4. Syntax to run the logistic regression model for Example 2

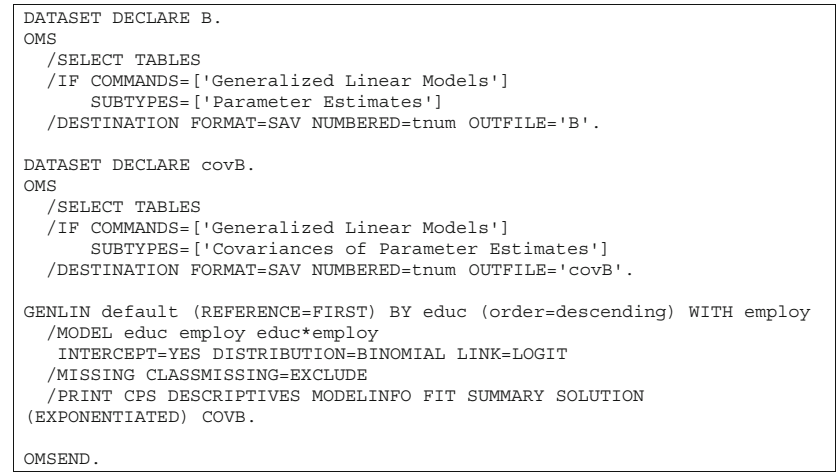

Table 2 shows the parameter estimates for this model, and Fig. 2 shows fitted values of the log-odds of defaulting on a 
Table 2 Regression coefficients for Example 2

Parameter Estimates

\begin{tabular}{|c|c|c|c|c|c|c|c|c|c|c|}
\hline \multirow[t]{2}{*}{ Parameter } & \multirow[t]{2}{*}{$\mathrm{B}$} & \multirow[t]{2}{*}{ Std. Error } & \multicolumn{2}{|c|}{ 95\% Wald Confidence Interval } & \multicolumn{3}{|l|}{ Hypothesis Test } & \multirow[t]{2}{*}{$\operatorname{Exp}(B)$} & \multicolumn{2}{|c|}{$\begin{array}{l}95 \% \text { Wald Confidence Interval } \\
\text { for } \operatorname{Exp}(\mathrm{B})\end{array}$} \\
\hline & & & Lower & Upper & Wald Chi-Square & df & Sig. & & Lower & Upper \\
\hline (Intercept) & -.449 & .2061 & -.853 & -.045 & 4.749 & 1 & .029 & .638 & .426 & .956 \\
\hline$[$ educ $=4]$ & .207 & .4910 & -.755 & 1.169 & .178 & 1 & .673 & 1.230 & .470 & 3.220 \\
\hline$[\mathrm{educ}=3]$ & .164 & .4137 & -.647 & .975 & .158 & 1 & .691 & 1.179 & .524 & 2.652 \\
\hline$[$ educ $=2]$ & .995 & .3456 & .318 & 1.672 & 8.288 & 1 & .004 & 2.705 & 1.374 & 5.325 \\
\hline$[\mathrm{educ}=1]$ & $0^{\mathrm{a}}$ & . & . & . & . & . & . & 1 & . & . \\
\hline employ & -.109 & .0238 & -.156 & -.062 & 20.929 & 1 & .000 & .897 & .856 & .940 \\
\hline$[\text { educ }=4]^{*}$ employ & .029 & .0731 & -.114 & .172 & .156 & 1 & .693 & 1.029 & .892 & 1.188 \\
\hline$[\text { educ }=3]^{*}$ employ & .062 & .0449 & -.026 & .150 & 1.896 & 1 & .169 & 1.064 & .974 & 1.162 \\
\hline$[\text { educ }=2]^{*}$ employ & -.128 & .0518 & -.230 & -.027 & 6.116 & 1 & .013 & .880 & .795 & .974 \\
\hline $\begin{array}{l}{[\text { educ }=1]^{*} \text { employ }} \\
(\text { Scale })\end{array}$ & $\begin{array}{l}0^{\mathrm{a}} \\
1^{\mathrm{b}}\end{array}$ & . & . & . & . & . & . & 1 & . & . \\
\hline
\end{tabular}

Dependent Variable: Previously defaulted

Model: (Intercept), educ, employ, educ * employ

${ }^{a}$ Set to zero because this parameter is redundant.

${ }^{\mathrm{b}}$ Fix at the displayed value.

loan as a function of the two explanatory variables and their interaction. One drawback to GENLIN is that it does not report the multiple degree of freedom Wald tests for EDUC and the EDUC $\times$ EMPLOY interaction. The LOGISTIC REGRESSION procedure, on the other hand, does include those multiple degree of freedom Wald tests in its table of regression coefficients. For EDUC, Wald = 8.577, $d f=3, p=.035$, and for EDUC*EMPLOY, Wald $=$ $10.519, d f=3, p=.015$.

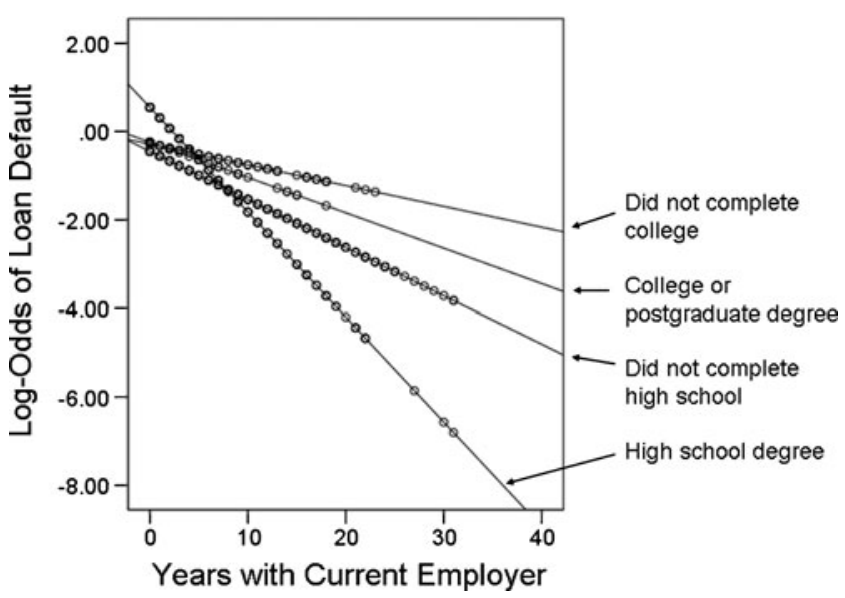

Fig. 2 The log-odds of loan default as a function of years with current employer and education level. The circles indicate combinations of the two explanatory variables that exist in the data file
One strategy that could be used to illustrate the nature of the interaction would be to compare fitted values for several combinations of EDUC and EMPLOY to a common reference point. In this example, the reference point EDUC $=1$ (high school not completed) and EMPLOY = 0 (0 years with current employer) was chosen. Fitted values for the following combinations of EDUC and EMPLOY were then compared with that common reference point:

- $\quad$ EDUC $=1$ (did not complete high school) and EMPLOY $=10$

- $\quad \mathrm{EDUC}=2$ (high school degree) and EMPLOY $=10$

- $\quad \mathrm{EDUC}=3$ (some college) and EMPLOY $=10$

- $\quad \mathrm{EDUC}=4$ (college or postgrad degree) and EMPLOY $=10$.

Before the !MLEcomp macro could be used to make those comparisons, some data management steps had to be carried out on the "B" and "covB" data sets created via GENLIN and OMS. The details of those data management steps are not described in detail here, but the result can be seen in Fig. $3 .{ }^{16}$ Note that the regression coefficients are in variable B (as required by !MLEcomp) and that the covariance matrix for the coefficients is in variables Intercept to educ2employ.

\footnotetext{
${ }^{16}$ Syntax file Example_2.SPS, which is available as part of the online Supplementary Material, shows how the data management steps were carried out.
} 
Syntax Box 5. !MLEcomp commands to carry out the desired comparisons for the logistic regression model in Example 2

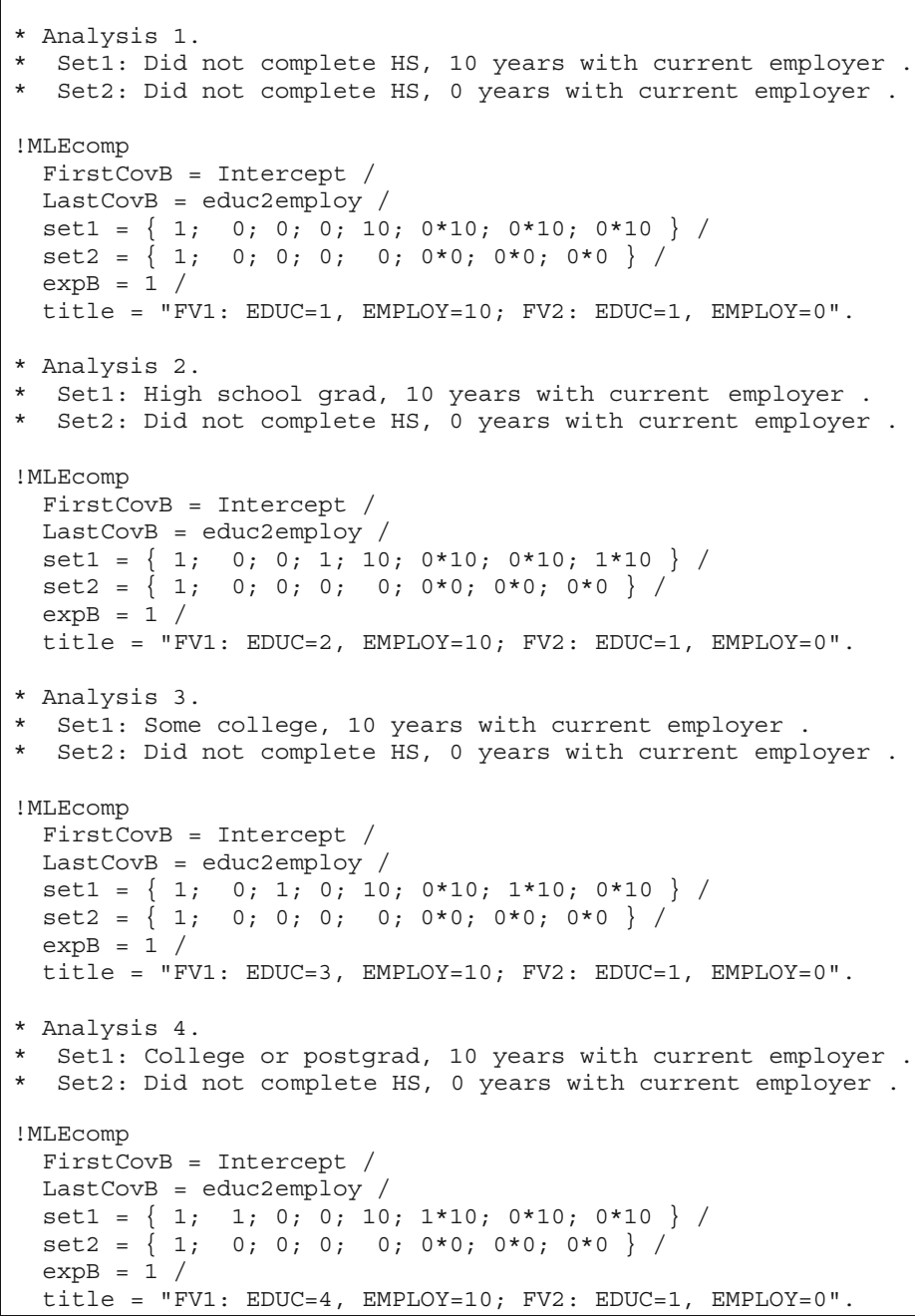

Syntax Box 5 shows !MLEcomp commands to carry out the four analyses described above. Lines that begin with an asterisk are comments. The first value in the Set1 and Set2 matrices contains an indicator for inclusion of the intercept ( $1=$ include, $0=$ exclude $)$ and must be set to 1 . The other seven values in Set1 and Set 2 are for educ $=4$, educ $=3$, educ $=2$, employ, [educ $=4]^{*}$ employ, [educ $\left.=3\right]^{*}$ employ, and [educ $=2]^{*}$ employ. Note that these are in the same order as in the table of parameter estimates from GENLIN (see Table 2), which is also the same order as in the covariance matrix for the parameter estimates. ${ }^{17}$ It is also important to note that the redundant variables (educ $=1$ and [educ $=1$ ] *employ) are not represented in Set1 and Set2. ${ }^{18}$

Finally, the ExpB argument is set to 1 in each case in order to dislay $\operatorname{Exp}(\mathrm{B})$ and its $95 \%$ confidence interval in the output from !MLEcomp. For a logistic regression model, $\operatorname{Exp}(\mathrm{B})$ provides an odds ratio.

For the sake of brevity, we will show the results for only the fourth analysis (see Output Box 3). Results for the first three analyses can be obtained by running syntax file Example 2.sps, which is available as part of the online Supplementary Material.

\footnotetext{
${ }^{17}$ The category numbers for EDUC appear in descending order because of the "order = descending" option we included in the GENLIN command. That was done in order to make EDUC $=1$ (high school not completed) the reference category.
}

\footnotetext{
${ }^{18}$ The redundant variables represent the reference categories for EDUC and EDUC*EMPLOY.
} 
Output Box 3. Results for Example 2, Analysis 4

\begin{tabular}{|c|c|c|c|c|c|c|c|c|c|}
\hline \multicolumn{10}{|c|}{ Run MATRIX procedure: } \\
\hline \multicolumn{10}{|c|}{ FV1: $E D U C=4, E M P L O Y=10 ; \quad F V 2: E D U C=1, E M P L O Y=0$} \\
\hline Set 1 & 1.00 & 1.00 & .00 & .00 & 10.00 & 10.00 & .00 & .00 & \\
\hline Set2 & 1.00 & .00 & .00 & .00 & .00 & .00 & .00 & .00 & \\
\hline S1-S2 & .00 & 1.00 & .00 & .00 & 10.00 & 10.00 & .00 & .00 & \\
\hline S2-S1 & .00 & -1.00 & .00 & .00 & -10.00 & -10.00 & .00 & .00 & \\
\hline FV1: EDU & $\begin{array}{r}=4, \quad \mathrm{EMPI} \\
\mathrm{B}\end{array}$ & $\begin{array}{r}\mathrm{OY}=10 ； \mathrm{FV} \\
\mathrm{SE}\end{array}$ & $\begin{array}{l}\text { EDUC= } \\
\text { Lower }\end{array}$ & $\begin{array}{l}\text { EMPLOY } \\
\text { Upper }\end{array}$ & Wald & $\mathrm{p}$ & $\operatorname{Exp}(B)$ & Lower & Upper \\
\hline FV1 & -1.043 & .505 & -2.033 & -.052 & 4.258 & .039 & .353 & .131 & .949 \\
\hline FV2 & -.449 & .206 & -.853 & -.045 & 4.749 & .029 & .638 & .426 & .956 \\
\hline FV1-FV2 & -.593 & .546 & -1.663 & .476 & 1.182 & .277 & .552 & .190 & 1.610 \\
\hline FV2-FV1 & .593 & .546 & -.476 & 1.663 & 1.182 & .277 & 1.810 & .621 & 5.275 \\
\hline
\end{tabular}

The fourth analysis compares people with a college or postgraduate degree (EDUC $=4$ ) who have been with their current employer for 10 years $($ EMPLOY $=$ 10) with the reference group for all analyses (EDUC = 1 and EMPLOY $=0$ ). One difference from the !OLScomp output shown earlier is that the $t$-tests have been replaced with Wald tests, which are customary for models fitted via MLE. Moreover, because the ExpB argument was set to 1 when invoking !MLEcomp, $\operatorname{Exp}(\mathrm{B})$ and its $95 \%$ confidence interval are shown in the output. The $\operatorname{Exp}(\mathrm{B})$ value on the FV1-FV2 line indicates that the odds of defaulting on a loan are .552 times lower for college graduates who have been with the same employer for 10 years (95\% CI, 0.190-1.610); or stated the other way, $\operatorname{Exp}(B)$ from the FV2-FV1 line tells us that the odds of defaulting are 1.810 times greater for those who have not completed high school and have just started working for their current employer (95\% CI, 0.621-5.275). Note, however, that in both cases, the confidence intervals include a value of 1 , which means that the odds ratios are not statistically significant at the .05 level $(p=.277)$.

\section{Example 3: Comparing two differences}

The first two examples have shown how the !OLScomp and !MLEcomp macros can be used to compare two fitted values from the same regression model. However, !OLScomp

and !MLEcomp can also be used to examine differences of differences. The linear regression model from Example 1 (MPG as a function of vehicle weight) can be used to illustrate.

Recall that for the first analysis in Example $1(3,000$ lbs-2,000 lbs), the FV1 - FV2 difference was -10.081 ; and for the third analysis $(5,000 \mathrm{lbs}-4,000 \mathrm{lbs})$, the FV1 - FV2 difference was -3.085 . The difference between those differences can be analyzed by making Set $_{1}=\mathrm{S} 1$ - S2 from the first analysis and $\mathrm{Set}_{2}=\mathrm{S} 1-\mathrm{S} 2$ from the third analysis. The !OLScomp command to do this is shown in Syntax Box 6, and the results in Output Box 4. The first point to note is that the results for FV1 and FV2 in this analysis match exactly the FV1-FV2 results from first and third analyses. Second, note that the difference between -10.081 and -3.085 - that is, the difference between the differences-is -6.996 , with the standard error and $95 \%$ confidence interval as shown.

Syntax Box 6. Using !OLScomp to examine a difference of differences

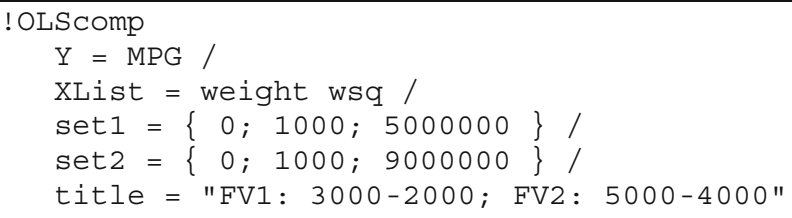


Output Box 4. !OLScomp results for a difference of differences

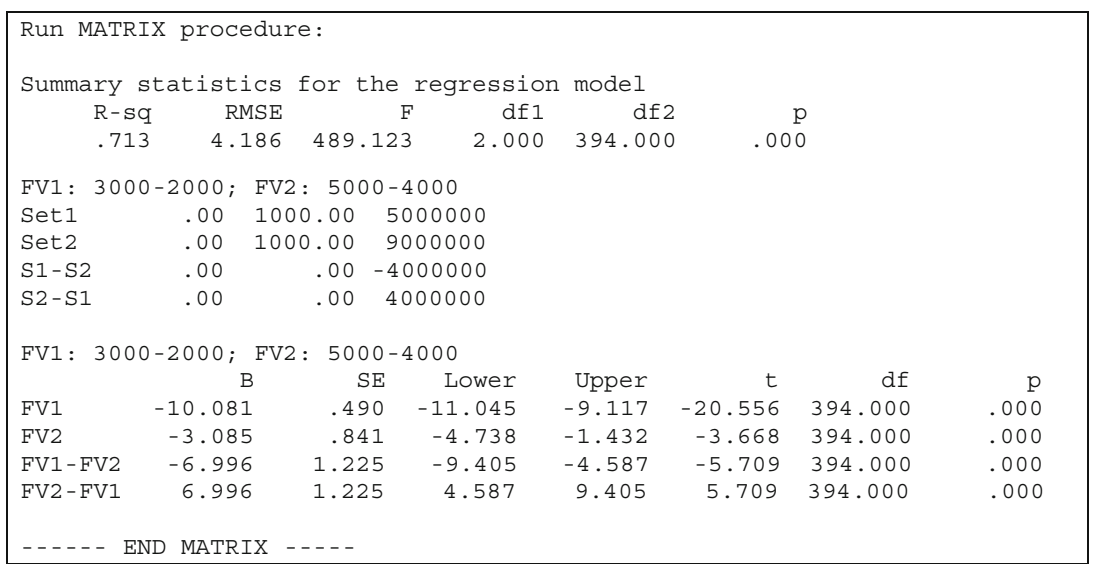

Differences of differences can be analyzed in the same fashion using !MLEcomp, as shown in syntax file MLEcomp_validation.SPS, which is available as part of the online Supplementary Material. Details are not provided here, but note that when the results are exponentiated, a difference of differences becomes a ratio of ratios. In the case of logistic regression, for example, it would be a ratio of odds ratios.

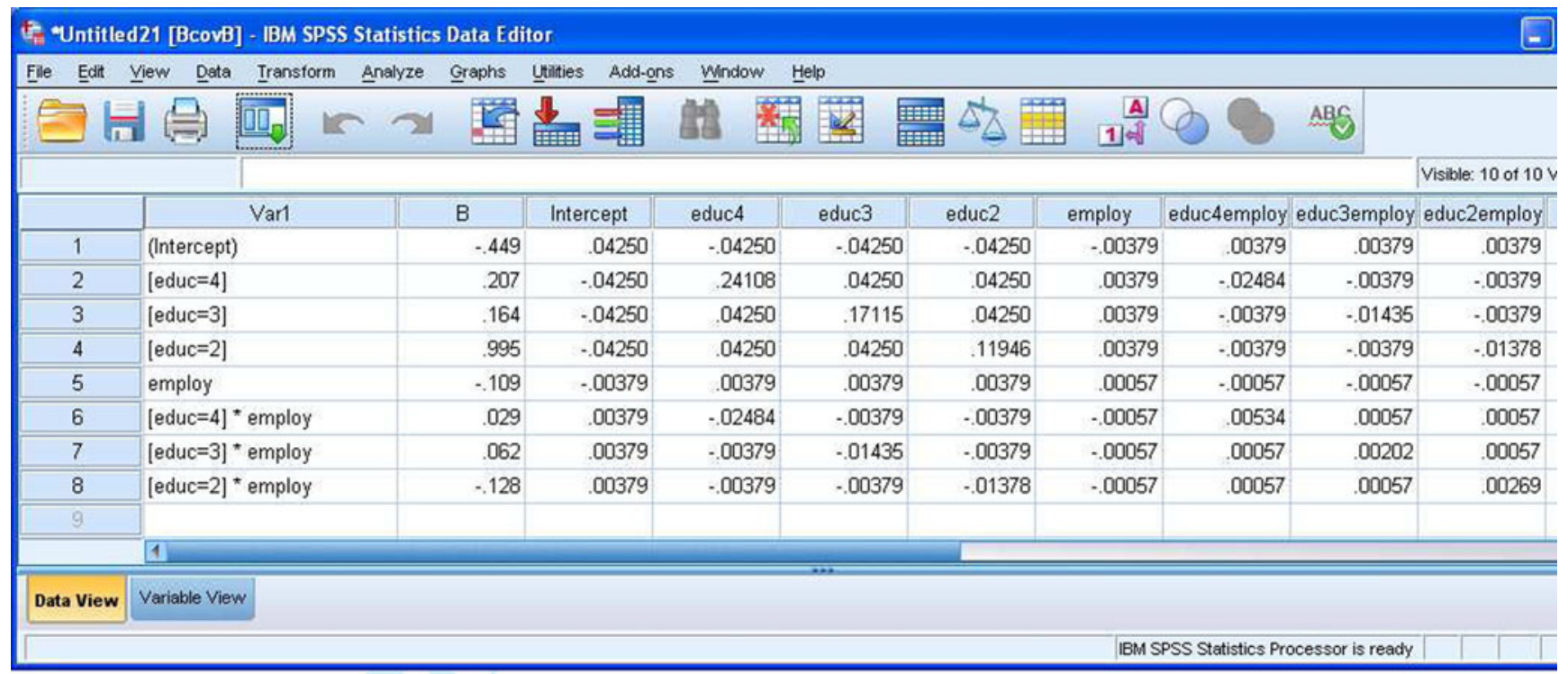

Fig. 3 Data set containing the vector of regression coefficients (variable B) and the covariance matrix for the coefficients (variables Intercept to educ2employ) for Example 2 


\section{Validation of the macros}

To validate the macros, we ran models that have only categorical explanatory variables and then compared results from the !OLScomp and !MLEcomp macros with results for the same fitted value comparisons obtained via estimated marginal means (EMMEANS) with pairwise comparisons. The macros were validated in all cases. Interested readers can find a detailed report of the validation analyses in Appendix 2.

\section{Summary}

When one fits a regression model, there is always interest in comparing fitted values of the outcome variable for different combinations of the explanatory variables. Some of those fitted value comparisons may be captured by single regression coefficients. When that is the case, the standard error needed for computing a confidence interval or for carrying out a statistical test on the difference is available in the regression output. However, other fitted value comparisons of interest may not be captured by a single regression coefficient. Rather, they are captured by the weighted combination of two or more regression coefficients. In this case, the standard error needed to make the fitted value comparison does not appear in the regression output. But the !OLScomp and !MLEcomp macros described in this article can be used to compare any two fitted values from regression models fitted by OLS and MLE, respectively. The output from both macros includes the difference between the two fitted values, its standard error, a $95 \%$ confidence interval on the difference, and a statistical test of the null hypothesis that the difference equals zero.

Acknowledgments We thank Hillary Maxwell, Mary Lou Schmuck, and Michel Bédard for helpful feedback on earlier drafts of the manuscript. We also thank Marta García-Granero for providing SPSS matrix language code to compute area under the $t$-distribution. Finally, we acknowledge the helpful contributions of two anonymous reviewers.

Competing interests None of the authors have any competing interests.

\section{Appendix 1 Computation confidence intervals and statistical tests}

Ninety-five percent confidence intervals are computed as point estimate \pm a critical value times the standard error of the point estimate.

- $95 \% \mathrm{CI}$ for $\mathrm{FV}_{1}=\mathrm{FV}_{1} \pm$ critical value $\times S E\left(\mathrm{FV}_{1}\right)$

- $95 \% \mathrm{CI}$ for $\mathrm{FV}_{2}=\mathrm{FV}_{2} \pm$ critical value $\times S E\left(\mathrm{FV}_{2}\right)$
- $95 \% \mathrm{CI}$ for $\left(\mathrm{FV}_{1}-\mathrm{FV}_{2}\right)=\left(\mathrm{FV}_{1}-\mathrm{FV}_{2}\right) \pm$ critical value $\times$ $\operatorname{SE}\left(\mathrm{FV}_{1}-\mathrm{FV}_{2}\right)$

- $95 \% \mathrm{CI}$ for $\left(\mathrm{FV}_{2}-\mathrm{FV}_{1}\right)=\left(\mathrm{FV}_{2}-\mathrm{FV}_{1}\right) \pm$ critical value $\times$ $\operatorname{SE}\left(\mathrm{FV}_{2}-\mathrm{FV}_{1}\right)$

For regression models fitted via MLE, the critical value is 1.96 , which is the 97.5 th percentile of the standard normal distribution. For models fitted via OLS, the critical value is the 97.5 th percentile of the $t$-distribution with degrees of freedom equal to the error degrees of freedom from the regression model. (The error degrees of freedom for an OLS model are equal to the sample size minus the total number of model parameters, including the constant.)

Statistical tests are also carried out. For models fitted via MLE, Wald tests are computed as follows:

- Wald $_{1}=$ Wald test value for $\mathrm{FV}_{1}=\left(\mathrm{FV}_{1} / \operatorname{SE}\left(\mathrm{FV}_{1}\right)\right)^{2}$

- $\mathrm{Wald}_{2}=\left(\mathrm{FV}_{2} / \mathrm{SE}\left(\mathrm{FV}_{2}\right)\right)^{2}$

- Wald $_{1-2}=\left(\left(\mathrm{FV}_{1}-\mathrm{FV}_{2}\right) / S E\left(\mathrm{FV}_{1}-\mathrm{FV}_{2}\right)\right)^{2}$

- $\mathrm{Wald}_{2-1}=\left(\left(\mathrm{FV}_{2}-\mathrm{FV}_{1}\right) / S E\left(\mathrm{FV}_{2}-\mathrm{FV}_{1}\right)\right)^{2}$.

And for models fitted via OLS, $t$-tests are computed as follows:

$$
\begin{array}{ll}
\text { - } & t_{1}=\mathrm{FV}_{1} / S E\left(\mathrm{FV}_{1}\right) \\
\text { - } & t_{2}=\mathrm{FV}_{2} / S E\left(\mathrm{FV}_{2}\right) \\
\text { - } & t_{1-2}=\left(\mathrm{FV}_{1}-\mathrm{FV}_{2}\right) / S E\left(\mathrm{FV}_{1}-\mathrm{FV}_{2}\right) \\
\text { - } & t_{2-1}=\left(\mathrm{FV}_{2}-\mathrm{FV}_{1}\right) / S E\left(\mathrm{FV}_{2}-\mathrm{FV}_{1}\right) .
\end{array}
$$

Note that because of the squaring, Wald $_{1-2}$ and Wald ${ }_{2-1}$ will have exactly the same value. For OLS models, $t_{1-2}$ and $t_{2-1}$ will have the same magnitude but opposite signs. The null hypothesis for all of these tests is that the parameter being tested (i.e., $\mathrm{FV}_{1}, \mathrm{FV}_{2}$, or the difference between them) equals zero. When the null hypothesis for a Wald test is true, the test value is distributed as chi-square with one degree of freedom $(d f)$. When the null hypothesis for a $t$-test is true, the test value is distributed as $t$ with $d f=d f_{\text {error }}$ from the regression model, which is equal to the sample size minus the number of parameters estimated by the model (including the constant).

Finally, for certain models fitted via MLE, it may be desirable to exponentiate the fitted values and their confidence intervals. For logistic regression, for example, $e^{B}$, which can also be written as $\operatorname{Exp}(B)$, equals the odds ratio. For Poisson regression, $\operatorname{Exp}(B)$ gives the rate ratio, sometimes called the incidence rate ratio.

\section{Appendix 2 Validation of the macros}

To validate !OLScomp, we ran a $2 \times 2$ ANOVA model using data from the Employee data.sav file that comes with SPSS. However, our OLScomp_validation.sps syntax file actually uses a copy of that data file that was renamed to Employee data.sav, with an underscore character (" ") between Employee and data. 
Table 3 Cell means from the $2 \times 2$ ANOVA model

\section{Estimates}

Dependent Variable:Salary (in thousands dollars)

\begin{tabular}{llllll}
\hline Gender & Minority Classification & Mean & Std. Error & & \multicolumn{2}{l}{$95 \%$ Confidence Interval } \\
\cline { 4 - 6 } & & & & Lower Bound \\
\hline Female & No & 26.707 & 1.112 & 24.521 & Upper Bound \\
& Yes & 23.062 & 2.333 & 18.477 & 28.893 \\
Male & No & 44.475 & 1.060 & 42.393 & 36.558 \\
& Yes & 32.246 & 1.845 & 28.621 & 35.871 \\
\hline
\end{tabular}

This was done because when a file with spaces embedded in its name is downloaded from a Web site, the spaces are often replaced with underscore characters. Therefore, we inserted the underscore character to ensure that the file name after download matches the file name we use in syntax file OLScomp_validation.sps.

The outcome variable for this validation analysis was salary (in thousands of dollars), and the two between-subjects factors were male gender $(1=$ male, $0=$ female $)$ and minority status $(1=$ yes, $0=$ no $)$. The male $\times$ minority interaction was also included. The most common way to run this model in SPSS is with the UNIANOVA procedure, although one could obtain exactly the same model using the REGRESSION procedure. One advantage of UNIANOVA is that it can generate a table of estimated marginal means (EMMEANS) for the interaction term, including pairwise comparisons that give the simple main effects of one factor at each level of the other factor (e.g., the yes - no difference for minority within males and females separately). SyntaxBox 7 shows the UNIANOVA syntax we used to run the model and generate the tables of simple main effects for both gender and minority.

The UNIANOVA results showed that $R^{2}=.258, M S_{\text {error }}=$ 217.804 , and $F(3,470)=54.405, p<.001{ }^{19}$ The cell means and the pairwise comparisons generated by the UNIANOVA syntax are shown in Tables 3, 4 and 5.

Syntax Box 7. Syntax to run a $2 \times 2$ ANOVA and generate tables of estimated marginal means with pairwise comparisons

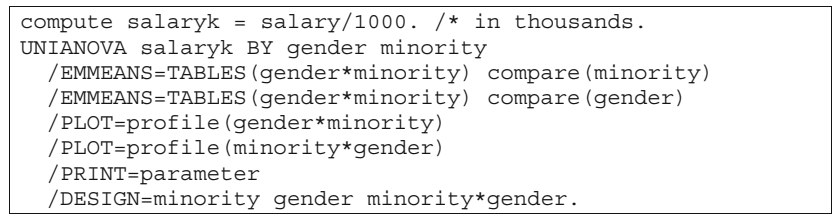

\footnotetext{
${ }^{19}$ This is the omnibus $F$-test, or the $F$-test for the "corrected model," as it says in the UNIANOVA output.
}

To make those same pairwise comparisons with the !OLScomp macro, we must first ensure that all categorical variables are coded as indicator variables, and then we must compute the product term needed for the interaction. As it happens, the gender variable in the raw data file is a string with values " $\mathrm{f}$ " and " $\mathrm{m}$." Therefore, we recoded it into an indicator variable called "male" $(1=$ male, $0=$ female). The minority variable was already a 1-0 indicator variable, so there was no need to recode it. Syntax to compute the needed variables and to call !OLScomp to make the desired pairwise comparisons is shown in Syntax Box 8. Output from those !OLScomp runs is shown in Output Box 5.

Syntax Box 8. Syntax to compute the needed indicator and product variables and !OLScomp commands to make the desired pair-wise comparisons

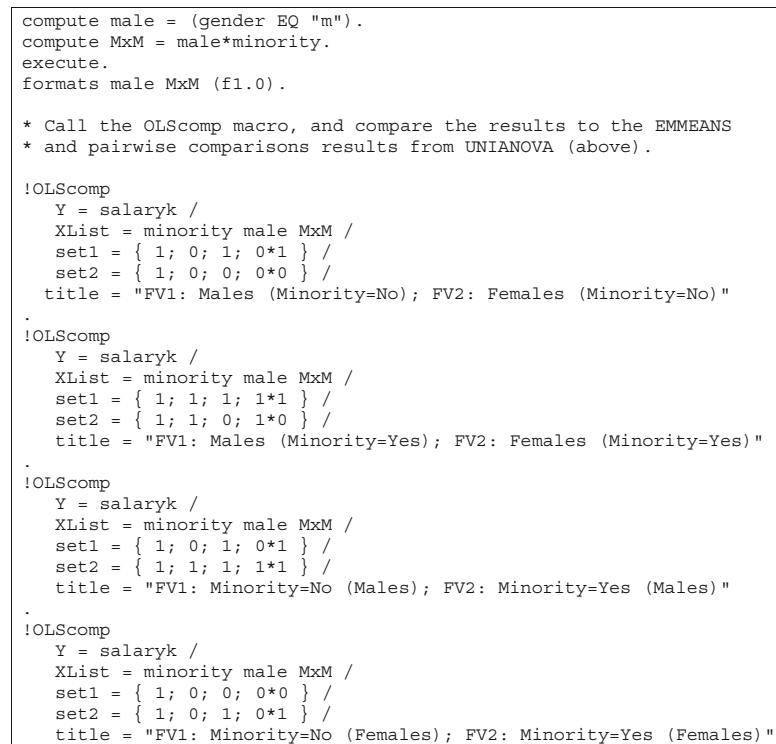
title = "FV1: Minority=No (Females); FV2: Minority=Yes (Females)" 
Output Box 5. !OLScomp results showing the same pairwise comparisons generated via EMMEANS using UNIANOVA

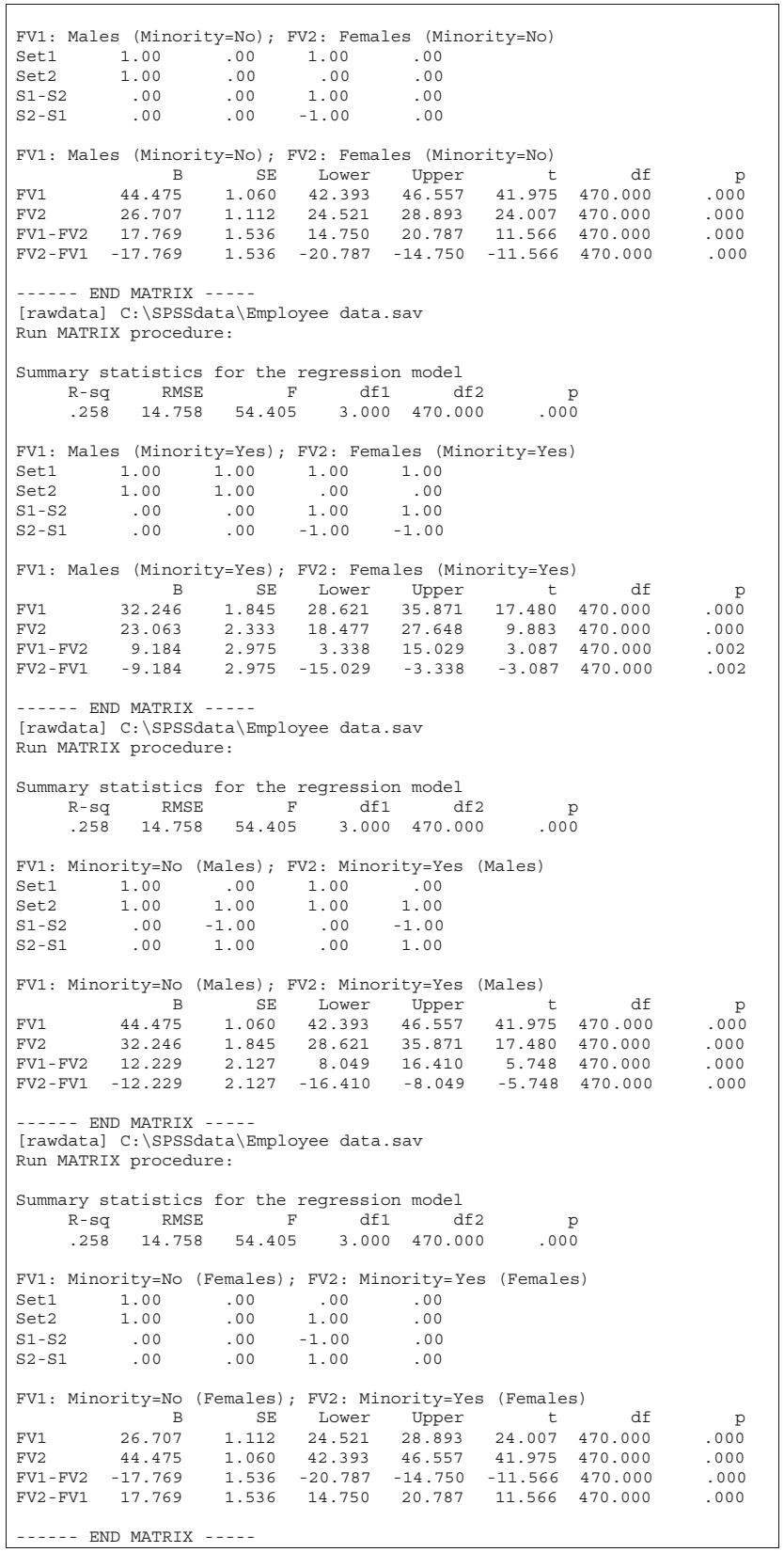

As usual, the output from !OLScomp shows the $R^{2}$ value for the model, the root mean square error (RMSE), and the omnibus $F$-test. The $R^{2}$ and $F$ values match exactly what we found when we ran the analysis via UNIANOVA, and apart from a little rounding error, the square of the RMSE =
$M S_{\text {error }}$ from UNIANOVA, so the user can be confident that !OLScomp is producing the same model as UNIANOVA.

Turning to the results for the first call to !OLScomp (see Output Box 5), note that FV1 $=44.475$ and FV2 $=26.707$. These are the mean salaries (in thousands of dollars) for males and females for whom minority status $=$ no. Note that they agree exactly with the EMMEANS produced via UNIANOVA (see Table 3). Note too that the FV1 - FV2 difference agrees exactly with the male-female pairwise comparison generated via UNIANOVA, right down to the standard error and $95 \%$ confidence interval (see Table 5). The results for the other three calls to !OLScomp in Syntax Box 8 can be verified in similar fashion.

One further way to validate !OLScomp is to test whether it gives correct results for the difference between two differences. Specifically, can it give us the $\mathrm{M}-\mathrm{F}$ difference when minority = yes minus the $\mathrm{M}-\mathrm{F}$ difference when minority $=$ no?

As we saw earlier, programming !OLScomp to give a difference between two differences is very straightforward if one has already used it for looking at the two primary differences one now wishes to compare. In that case, the values that need to be entered in the Set 1 and Set 2 vectors can be read directly from earlier output. For the example we are considering, the two primary differences are M-F (Minority $=$ Yes $)$ and $\mathrm{M}-\mathrm{F}($ Minority $=$ No). We previously used !OLScomp to examine both of them. Here are the relevant parts of the output from those analyses:

$\begin{array}{lcccc}\text { FV1: Males (Minority=Yes) ; FV2: Females (Minority=Yes) } \\ \text { Set1 } & 1.00 & 1.00 & 1.00 & 1.00 \\ \text { Set2 } & 1.00 & 1.00 & .00 & .00 \\ \text { S1-S2 } & .00 & .00 & 1.00 & 1.00 \\ \text { FV1: Males (Minority=No) ; FV2: Females (Minority=No) } \\ \text { Set1 } & 1.00 & .00 & 1.00 & .00 \\ \text { Set2 } & 1.00 & .00 & .00 & .00 \\ \text { S1-S2 } & .00 & .00 & 1.00 & .00\end{array}$

The S1-S2 line from the M-F (Minority = Yes) output gives us the values we now need to enter as $\mathrm{Set}_{1}$; and the $\mathrm{S} 1-\mathrm{S} 2$ line from the M-F (Minority $=$ No) output gives us the values we now need to enter as Set $_{2}$. Therefore, we must call !OLScomp as shown in Syntax Box 9. Output from that call is shown in Output Box 6.

Syntax Box 9. !OLScomp syntax to compare the M - F differences for minority and nonminority cases

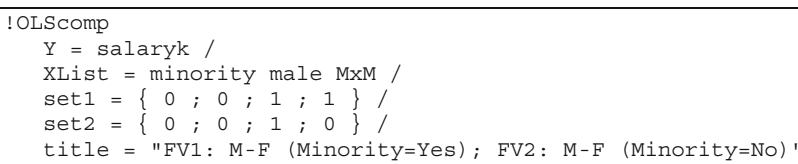


Table 4 Simple main effects of minority classification for females and males

Pairwise Comparisons

Dependent Variable:Salary (in thousands of dollars)

\begin{tabular}{|c|c|c|c|c|c|c|c|}
\hline \multirow[t]{2}{*}{ Gender } & \multirow{2}{*}{$\begin{array}{l}\text { (I) Minority } \\
\text { Classification }\end{array}$} & \multirow{2}{*}{$\begin{array}{l}\text { (J) Minority } \\
\text { Classification }\end{array}$} & \multirow[t]{2}{*}{ Mean Difference (I-J) } & \multirow[t]{2}{*}{ Std. Error } & \multirow[t]{2}{*}{ Sig. ${ }^{a}$} & \multicolumn{2}{|c|}{$95 \%$ Confidence Interval for Difference } \\
\hline & & & & & & Lower Bound & Upper Bound \\
\hline \multirow[t]{2}{*}{ Female } & No & Yes & 3.644 & 2.585 & .159 & -1.435 & 8.724 \\
\hline & Yes & No & -3.644 & 2.585 & .159 & -8.724 & 1.435 \\
\hline \multirow[t]{2}{*}{ Male } & No & Yes & $12.229 *$ & 2.127 & .000 & 8.049 & 16.410 \\
\hline & Yes & No & $-12.229 *$ & 2.127 & .000 & -16.410 & -8.049 \\
\hline
\end{tabular}

Based on estimated marginal means

a. Adjustment for multiple comparisons: Least Significant Differene (equivalent to no adjustment).

*. The mean difference is significant at the .050 level

Table 5 Simple main effects of sex within the two levels of minority classification

Pairwise Comparisons

Dependent Variable:Salary (in thousands of dollars)

\begin{tabular}{|c|c|c|c|c|c|c|c|}
\hline \multirow{2}{*}{$\begin{array}{l}\text { Minority } \\
\text { Classification }\end{array}$} & \multirow[t]{2}{*}{ (I) Gender } & \multirow[t]{2}{*}{ (J) Gender } & \multirow[t]{2}{*}{ Mean Difference (I-J) } & \multirow[t]{2}{*}{ Std. Error } & \multirow[t]{2}{*}{ Sig. ${ }^{a}$} & \multicolumn{2}{|c|}{$95 \%$ Confidence Interval for Difference } \\
\hline & & & & & & Lower Bound & Upper Bound \\
\hline \multirow[t]{2}{*}{ No } & Female & Male & $-17.769 *$ & 1.536 & .000 & -20.787 & -14.750 \\
\hline & Male & Female & $17.769 *$ & 1.536 & .000 & 14.750 & 20.787 \\
\hline \multirow[t]{2}{*}{ Yes } & Female & Male & $-9.184 *$ & 2.975 & .002 & -15.029 & -3.338 \\
\hline & Male & Female & $9.184 *$ & 2.975 & .002 & 3.338 & 15.029 \\
\hline
\end{tabular}

*. The mean difference is significant at the .050 level.

a. Adjustment for multiple comparisons:Least Significant Difference (equivalent to no adjustments)

Output Box 6. Output generated by the !OLScomp command shown in Syntax Box 9

\begin{tabular}{|c|c|c|c|c|c|c|}
\hline \multicolumn{7}{|c|}{ Run MATRIX procedure: } \\
\hline \multicolumn{7}{|c|}{ Summary statistics for the regression model } \\
\hline R-sq & RMSE & F df 1 & df2 & \multirow{2}{*}{\multicolumn{2}{|c|}{$\begin{array}{r}p \\
.000\end{array}$}} & \\
\hline .258 & 14.758 & 54.405 & 470.000 & & & \\
\hline \multicolumn{7}{|c|}{ FV1: M-F (Minority=Yes); FV2: M-F (Minority=No) } \\
\hline Seti & .00 & $.00 \quad 1.00$ & 1.00 & & & \\
\hline Set2 & .00 & 1.00 & .00 & & & \\
\hline $\mathrm{S} 1-\mathrm{s} 2$ & .00 & .00 & 1.00 & & & \\
\hline S2-S1 & .00 & .00 & -1.00 & & & \\
\hline \multicolumn{7}{|c|}{ FV1: M-F (Minority=Yes); FV2: M-F (Minority=No) } \\
\hline & B & SE Lower & Upper & $t$ & $d f$ & $\mathrm{p}$ \\
\hline FV1 & 9.184 & 3.338 & 15.029 & 3.087 & 470.000 & .002 \\
\hline FV2 & 17.769 & 14.750 & 20.787 & 11.566 & 470.000 & .000 \\
\hline FV1-FV2 & -8.585 & -15.164 & -2.006 & -2.564 & 470.000 & .011 \\
\hline FV2 - FV1 & 8.585 & 2.006 & 15.164 & 2.564 & 470.000 & .011 \\
\hline
\end{tabular}


Table 6 Parameter estimates from the $2 \times 2$ ANOVA model run via command shown in Syntax Box 7

Parameter Estimates

Dependent Variable:Salary (in thousands of dollars)

\begin{tabular}{|c|c|c|c|c|c|c|}
\hline \multirow[t]{2}{*}{ Parameter } & \multirow[t]{2}{*}{ B } & \multirow[t]{2}{*}{ Std. Error } & \multirow[t]{2}{*}{$\mathrm{t}$} & \multirow[t]{2}{*}{ Sig. } & \multicolumn{2}{|c|}{$95 \%$ Confidence Interval } \\
\hline & & & & & Lower Bound & Upper Bound \\
\hline Intercept & 32.246 & 1.845 & 17.480 & .000 & 28.621 & 35.871 \\
\hline$[$ minority $=0]$ & 12.229 & 2.127 & 5.748 & .000 & 8.049 & 16.410 \\
\hline$[$ minority $=1]$ & $0^{\mathrm{a}}$ & . & . & . & . & . \\
\hline [gender $=\mathrm{f}]$ & -9.184 & 2.975 & -3.087 & .002 & -15.029 & -3.338 \\
\hline$[$ gender $=\mathrm{m}]$ & $0^{\mathrm{a}}$ & . & . & . & . & . \\
\hline$[$ gender $=\mathrm{f}] *[$ minority $=0]$ & -8.585 & 3.348 & -2.564 & .011 & -15.164 & -2.006 \\
\hline$[\text { gender }=\mathrm{f}]^{*}[$ minority $=1]$ & $0^{\mathrm{a}}$ & . & . & . & . & . \\
\hline$[\text { gender }=\mathrm{m}]^{*}[$ minority $=0]$ & $0^{\mathrm{a}}$ & . & . & . & . & . \\
\hline$[$ gender $=\mathrm{m}] *[$ minority $=1]$ & $0^{\mathrm{a}}$ & . & . & . & . & . \\
\hline
\end{tabular}

a.This parameter is set to zero because it is redundant

Note that the FV1 and FV2 values from this analysis match $\mathrm{F} 1-\mathrm{F} 2$ differences that we saw in previous analyses of the primary differences. FV1 from this analysis $=\mathrm{FV} 1-$ FV2 from the earlier "M-F (Minority = Yes)" analysis; and $\mathrm{FV} 2$ from this analysis $=\mathrm{FV} 1-\mathrm{FV} 2$ from the earlier "M-F $($ Minority = No)" analysis (see Output Box 5).

To further validate the !OLScomp macro, we can compare the FV1 - FV2 result from this analysis with some output in the table of parameter estimates generated by UNIANOVA (Table 6). Note that the results for the male $\times$ minority interaction (the $\mathrm{M} \times \mathrm{M}$ line in Table 6) match exactly the FV1 - FV2 results from !OLScomp. That is, the regression coefficient for $\mathrm{M} \times \mathrm{M}=-8.585(S E=3.348)$, matching exactly the FV1 - FV2 output from !OLScomp.

Finally, we have validated the !MLEcomp macro in exactly the same manner, using the GENLIN procedure to generate estimated marginal means with pairwise comparisons. Readers who are interested in the details can run syntax file MLEcomp_validation.sps, which is available as part of the online Supplementary Material. Note that MLEcomp_validation.sps does not require a raw data file, since the needed raw data are generated internally via the DATA LIST command in conjunction with the WEIGHT command.

\section{References}

Fox, J. (2008). Applied regression analysis and generalized linear models (2nd ed.). Los Angeles: Sage.

Johnson, R. A., \& Wichern, D. W. (2002). Applied Multivariate Statistical Analysis (5th ed.). Upper Saddle River: Prentice Hall. 